\title{
IoT System with Access Control Integrating RFID and Non-invasive Air Conditioning
}

\author{
Elton Nobre Morais \\ Computing Department Federal \\ Institute of Ceará Maracanaú \\ Campus, Brazil
}

\author{
Pedro Hericson M. Araújo \\ Computing Department Federal \\ Institute of Ceará Maracanaú \\ Campus, Brazil
}

\author{
Leonardo da Silva Costa \\ Computing Department Federal \\ Institute of Ceará Maracanaú \\ Campus, Brazil
}

\begin{abstract}
This article describes the tools and technologies used to manage an integrated system consisting of RFID access control and noninvasive infrared air conditioning. In this sense, was developed an IoT (Internet of Things) platform consisting of hardware - responsible for controlling the access of users through the recognition of an individual RFID tag, as well as the capture and transmission of infrared signals - and software - a Web system for monitoring and remote control. Was also developed web software to monitor access and register new users and environments, where it is possible to define user profiles besides the registration of activities in each site. As resources are usually limited in IoT systems, it is necessary to use application protocols that optimize the transmission between sensor nodes and gateways, as a result of this the Message Queue Telemetry Transport (MQTT) and Constrained Application Protocol (CoAP) protocols were used for communication between the RFID access control system (RACS), infrared air conditioning control (IACC) and the Web system. The proposed platform was tested and validated in a classroom.
\end{abstract}

\section{General Terms}

Internet of Things, Message Queue Telemetry Transport, Radio Frequency Identification.

\section{Keywords}

IoT, Esp8266, IR, MQTT, CoAP, RFID.

\section{INTRODUCTION}

Social responsibility as a strategy adopted by companies, aiming for the well-being of employees, has brought significant returns in their performance [14]. Currently, not only lamps and fans, but also, for the better quality of life, in the residential as well as the commercial and industrial area, air conditioners are widely used, seeking through the control of the air conditioning a work or leisure environment more comfortable. Therefore, this device is no longer an object of luxury to become indispensable equipment, as well as refrigerators, televisions and stoves [6]. As an example of growth in the use of these appliances, a research made by EPE 1

\footnotetext{
${ }^{1}$ Energy research company.
}

in Brazil estimates that ownership by air conditioner families has more than doubled between 2005 and 2017 in the residential sector and demand for electricity due to its use can grow at a rate of $5.4 \%$ per year [16]. All these devices, especially air conditioners, if not used properly can generate a great waste of energy. According to Almeida [3], the average monthly consumption in homes can reach up to $21 \%$ in the energy bill, considering an air conditioning of 10,000 BTU, bound daily for an interval of 8 hours, having a total consumption of approximately R $\$$ : 200,00 (Brazilian real). According to research conducted by ABESCO 2 on the Energy Efficiency Potential in Brazil from 2008 to 2016, the volume of lost energy in the last 3 years is greater than all of Itaipu's electricity production in 2016, this energy being which would be enough to supply a city of 533,000 inhabitants for one month, which could generate a saving of $R \$ 61.71$ billion [2]. Therefore, it's needed to monitor and control the use of electrical devices to avoid wasting energy.

Objects equipped with electronic components such as radio frequency identifiers (RFID) or BLE ${ }^{3}$ modules are widely used to create an automated data acquisition and monitoring systems [11]. The search for profitability, through the rationalization of processes, influences companies to know these new technologies and methods of improvement. Radio Frequency Identification (RFID) "is one of those rare technologies that 'change the world', which will force a reconsideration of many strategies in the value chain" [18]. The concept of radiofrequency identification that has been recently used for several industrial applications [28] has received great attention from researchers and is gaining more and more space in the world, growing exponentially in recent years. RFID tags have a low cost and can be goods, vehicles or any object that needs to be monitored [7].

\section{RELATED WORK}

Efforts made in home automation seek to offer new forms of interaction in equipment already present in homes as well as to provide comfort in handling them [20]. Applying concepts of home automation, besides being able to bring comfort, security and practicality, can become an ally in the reduction of unnecessary

\footnotetext{
${ }^{2}$ Brazilian Association of Energy Conservation Services Companies.

${ }^{3}$ Bluetooth Low Energy.
} 
expenses in resources as water and energy [8]. From a perspective of automation at universities or in government offices, the easy interaction between devices such as sensors, actuators, displays, surveillance cameras, vehicles provides a wide range of data for analysis, monitoring and safety [19].

In this sense, several projects have been developed in recent years. Piyare [24] proposed a control and monitoring system for lights, temperature and current sensors using a RESTful Web service on an Arduino Uno connected to the network using shield ethernet. He developed an application on the Android platform to manage the sensors and electronic devices.

Figueiredo [17] has built a supervised control and data acquisition system (SCADA) in which the commands sent to the embedded system are performed by a MATLAB application that provides SCADA systems with the ability to manage complex control algorithms called the SCADA-MATLAB platform.

Specifically for air-conditioning devices, Mercier [21] has developed a universal remote control system. The built-in system allows a person to record IR signals from an unregistered device. It uses the Arduino Uno microcontroller to send the IR commands and an application on the Android platform to control the equipment. Communication between devices occurs through Bluetooth Low Energy (BLE).

Araujo [4] has developed a low-cost RFID access control system using Raspberry pi and PIC18F2550 Microcontroller. The frequency of the tags the system recognizes is $125 \mathrm{Khz}$ containing 14 bytes of information that are handled and posted in a Google Database, where information can be accessed from anywhere in the world. A flowchart explains the entire operation of the system, such as registration and tag recognition.

There are some weaknesses in these related papers. Proposals do not offer an option for simultaneous equipment control. In the works carried out by Piyare [24] and Figueiredo [17] the use of ethernet cables for communication makes the equipment difficult to install. In Mercier's [21] thesis it is possible only a local control because the scope is restricted by the capacity of the communication technology used.

\section{MESSAGE QUEUE TELEMETRY TRANSPORT (MQTT)}

Disseminated for its reliability, the Message Queue Telemetry Transport (MQTT) protocol was developed for devices with limited resources [23], operating at the application layer in the network. The operation of this protocol occurs in a topic-based publication-subscription architecture, which works as follows; when a customer posts a message $\mathrm{M}$ for a topic $\mathrm{T}$, this message $\mathrm{M}$ is passed on to all subscribers of topic T. The underlying layers of Transmission Control Protocol (TCP) and IP are of fundamental importance to MQTT, as well as Hypertext Transfer Protocol (HTTP), it also uses them.

In MQTT there are three levels of Quality of Service (QoS), namely: QoS level 0, when acknowledgment of receipt is not required and the message is delivered at most once. QoS level 1, when acknowledgment of receipt is required and the message is delivered at most once. QoS level 2, which utilizes a four-way handshake mechanism to ensure delivery exactly once [26].

\section{CONSTRAINED APPLICATION PROTOCOL (COAP)}

Constrained Applications Protocol (CoAP) is a protocol intended to be used in the device's communication with limited resources and is present in the application layer. It is based on the REST architecture and supports the request-response model as HTTP. The publish-subscribe model can also be used by CoAP by using the GET method, but different from MQTT, CoAP uses the publisher and subscribe model based on the Universal Resource Identifier (URI) [25].

One of the central features of the CoAP protocol facility is to use UDP protocol instead of TCP. It defines a kind of "message layer" to retransmit lost packets. In addition, within the UDP packets a four-byte binary header is defined, followed by a sequence of options where each of these has a one-byte header, which can be expanded to two bytes if greater data values are required. Using this more optimized and objective approach, a header with a total size of 10 to 20 bytes for simple request results [9].

\section{IOT PLATFORM}

Looking for a cheap and easy to assemble solution that meets the design requirements, we chose NodeMCU for hardware construction (Figures 1 and 6 .

NodeMCU is a firmware and open-source development kit widely used in embedded projects [27]. The kit consists of an ESP8266 microcontroller that contains a 32-bit microprocessor with support for Wi-Fi connection, plus power and programming ports, 10 digital inputs and one analog [22].

The platform provides a web interface for control and communicates with the devices using Message Queue Telemetry Transport (MQTT) and Constrained Application Protocol (CoAP) protocols. Through it, users with permission can access the site with the RFID tag and control the air conditioner.

\subsection{Infrared Air Conditioning Control (IACC)}

It was built a remotely managed embedded system to control air conditioners by transmitting IR commands. The IACC system is non-invasive as it acts as a remote control, sending commands to the air conditioner through an infrared LED. The device is fixed with the emitting LED in front of the air conditioner to ensure the best signal reception. Being non-invasive brings some benefits to the user, such as the continuity of the air conditioner factory warranty, the diversification of compatible equipment, and the easy setup of the equipment, as it is only necessary to identify the correct wave frequency, which is obtained by the original remote control of the air conditioner. This data is read through the IACC infrared receiver and then stored at the time of initial setup.

The IACC hardware consists of three parts: microcontroller with access to the Wi-Fi network, transmission module and capture module [12], Fig 1 shows the internal scheme.

Was added an infrared receiver Fig 2 A to the embedded system to capture control signals. Buttons on the air conditioning remote 


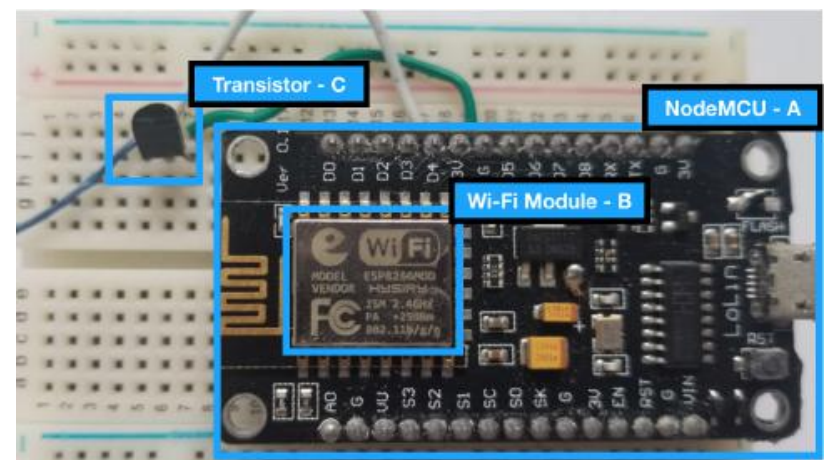

Fig. 1. IACC internal control scheme.

control were pressed so that the corresponding commands were stored and mapped for later use by the equipment, this data can be persisted on the server for other users to use. Fig 7 shows a prototype built for testing.

When receiving a request to execute a command, the embedded system uses the IACC to transmit the information to the equipment through an infrared command. The LEDs have been strategically distributed to achieve a larger coverage area to ensure better signal quality. Transistor BC 337 (Fig 1 -C) was incremented in the system to increase the signal strength and compensate for the loss of intensity due to the parallel connection of the LEDs.

The infrared emitting LEDs (Fig 2 -B) are responsible for sending the commands to the air conditioner. These commands must be previously stored in the initial IACC configuration. The process of reading the original remote control of the air conditioner is performed through the infrared receiver (Fig 2 $\mathrm{A}$ ), this reading is possible by pressing the button of the remote control while it is directed to the IACC. After being read, the commands are written for later use by the system.

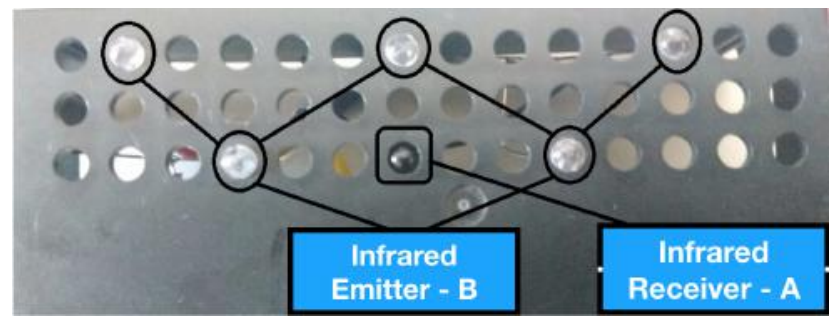

Fig. 2. IACC capture and transmition module.

One of the differentials of the IACC is monitoring the environment in which it will be installed, besides the low cost. The integration and communication with the RACS module makes it possible to create user profiles to create an environment configured according to the needs of the person who will enter the room.

An existing problem in a non-invasive solution is a possible inconsistency of the state of managed appliances resulting from manual control. Thus, to circumvent this exception, a temperature sensor was added. Therefore, coherent decisions and readjustments can be made by the system user or, in the future, by the system itself based on established standards in real time

\subsection{RFID Access Control System (RACS)}

The basic idea of radio frequency identification technology is to use a static electromagnetic inductor reader and a mobile microchip with an antenna, which can operate in $\mathrm{KHz}$ and in order of $\mathrm{MHz}$. This mobile microchip consists of a transponder or tag that does not need the power supply, since the signal that excites it comes directly from a reader-inductor circuit that can also be used for recording. Upon being excited, the mobile circuit is powered by sending or receiving recorded data [10].

One of the working principles of RFID technology is electromagnetic radiation, which is defined as being waves of electric and magnetic energy that are radiated together through space and, by radiation, the propagation of energy through space in the form of waves or particle [13].

A small part of the emitter field interacts with the transponder antenna coil with a certain distance from the reader coil. The magnetic induction generates a voltage in the transponder antenna coil. This voltage is rectified and serves as the power supply for the microchip. A capacitor is connected in parallel to the reader's antenna coil. The capacitance is selected to match the inductance of the antenna coil to form a parallel resonant circuit, i.e. to obtain a resonant frequency corresponding to the frequency of the reader transmission, the illustration is shown in Fig 3

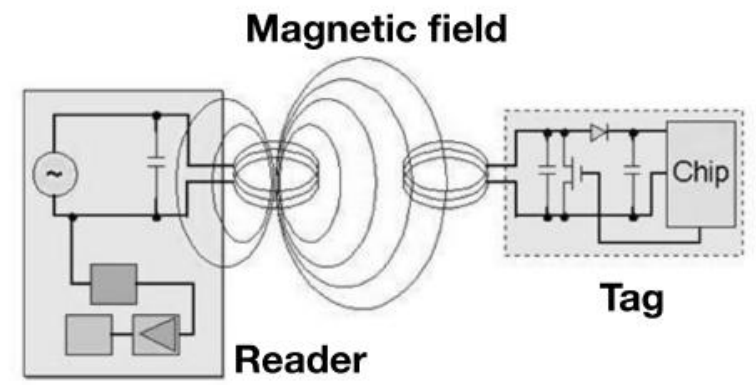

Fig. 3. Principle of inductive coupling [1].

\subsection{System Operation}

For the realization of the project was designed and built hardware composed of components such as ESP8266, Buzzer, Led, Relay, Transistor BC337, and LCD. The developed circuit is shown in Fig 4

In the operation of the proposed system with the ESP8266, the reader has a frequency of $13.56 \mathrm{MHz}$ that allows, for example, the reading of student, subway portfolios, used in Brazilian urban transport systems. The choice of the RFID receiver is associated with the ease of the students already possessing the tags to reduce the cost with the purchase of specific cards for the system. 


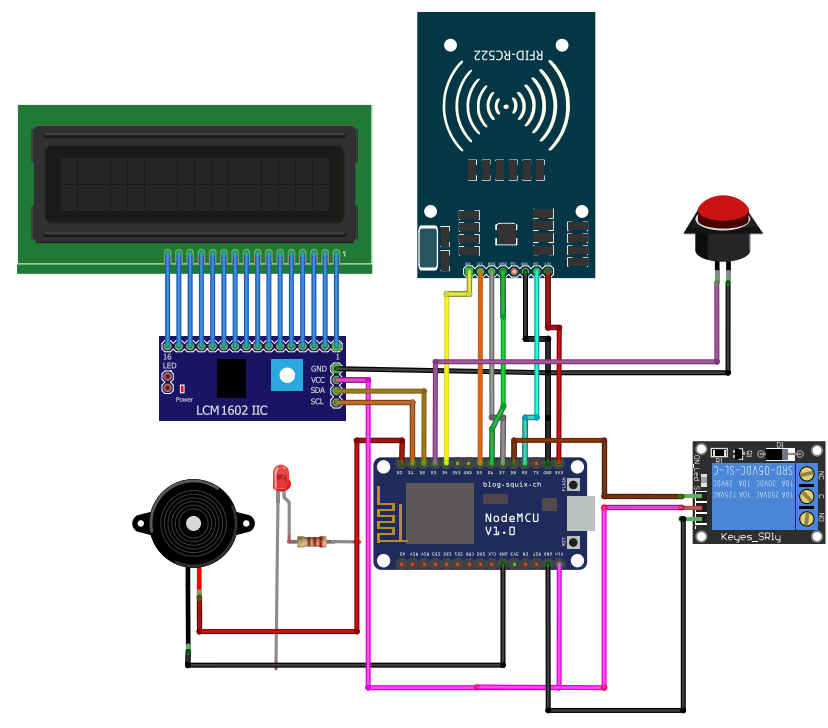

Fig. 4. Circuit developed [5].

The firmware is responsible only for reading tags and actuating the commands received from the back-end, the communication is made by the MQTT protocol and has data format in the standard JavaScript Object Notation (JSON).

\subsection{Integration of the IR system with RFID}

The proposed system integrates the functionalities of the RFID system with the IR system, called SmartRoom which aims to control classrooms in an automated and configurable way. All the architecture of the project is can be identified in Fig 5

It is possible to view in Fig 5 that the architecture of our proposal is based on the MQTT and CoAP protocols. In the RACS module, when bringing the card closer to the RFID reader the tag is recognized. This information is sent to the back-end through the MQTT_PUB using the MQTT Broker IoT Eclipse. The back-end returns a JSON, through MQTT_SUB with the user data and other related information. At this point, it is important to notice that this information is sent to both the RACS module and the IACC module.

When the MQTT Broker forwards the response with a JSON containing all information from the database to the RACS module, it extracts the relevant information and through the protocol I2C shows the data referring to the tag, at this moment the buzzer acts with a signal and the LED with a visual signal as feedback to users. Then the locked door is released for access to the environment. The IACC module extracts from JSON the information relevant to its actuators, including the preconfigured temperature for that environment.

The module sends the command to the infrared emitter to turn on the air conditioner, the LED flashes while the transmitter acts, since the frequency of the waves is imperceptible to the human eye.

In the IACC, the ambient temperature is often checked, when there is any change it is updated to the RACS module for the fidelity of

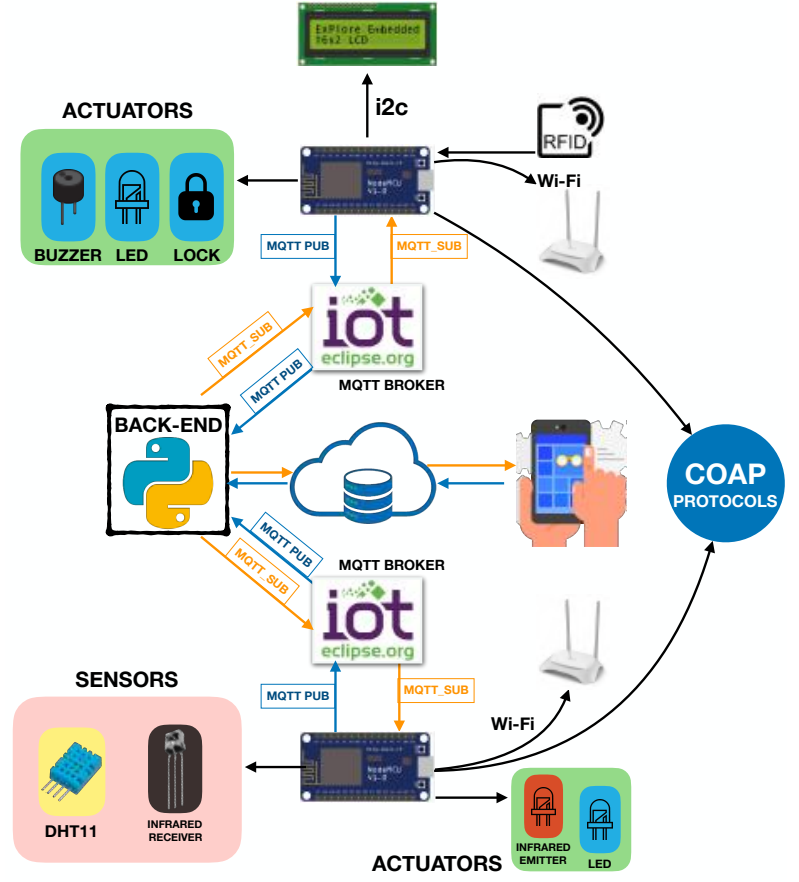

Fig. 5. IACC and RACS communication architecture via MQTT and CoAP.

the information in real-time. The CoAP protocol is responsible for sending the temperature obtained by the DHT11 sensor present in the IACC module directly to the RACS, without using the Internet.

Fig 6 -A and Fig 6-B, respectively, show the front and inner part of the system in a plastic box.

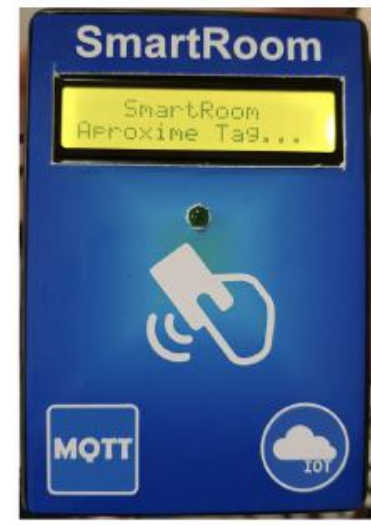

(A)

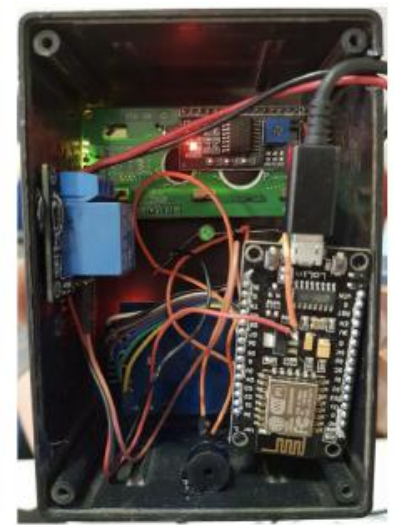

(B)
Fig. 6. Front and inner part of the system, respectively.

For the integration of the protists, the IACC was added, composed of an ESP8266 Microcontroller, DHT11 Temperature and Humidity Sensor, Infrared Emitter LED, Infrared Receiver and RGB Led, the prototype can be visualized in Fig 7 

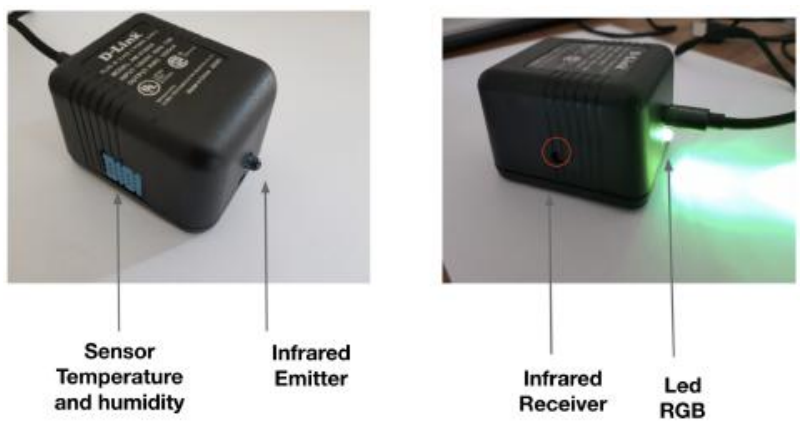

Fig. 7. IACC developed.

Fig 9 shows the flowchart containing all system features more intuitively, based on the settings previously established by the user through the web system developed with the Model-ViewController (MVC) design pattern.

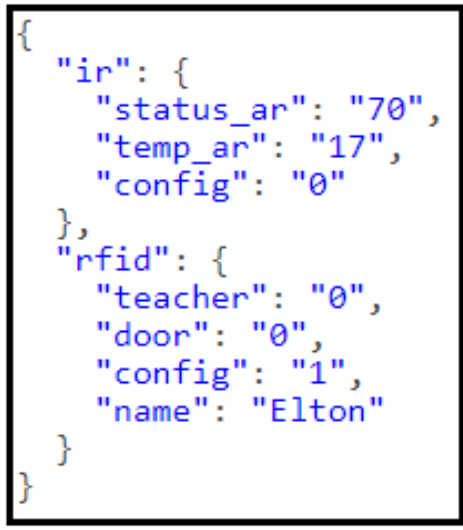

Fig. 8. JSON sent by back-end.

All control of the RACS and IACC actuations are performed through the information obtained from the back-end in JSON format, as shown in Fig 8

It is possible to notice the "ir" and "rfid" fields, because each device treats the information for its purpose, for example in "rfid" there is the field "name" because it will be displayed in the lcd display, since "ir" does not exist the necessity of this field. Already "temp_ar" sets the temperature of the air conditioning according to the configuration of the user in that environment. It is worth noticing that each user has a time allowed to access the room so the configuration will be disabled when the user brings his tag closer to RFID when leaving or when his time reaches 0 , whichever happens first will be activated.

\subsection{Web System}

The proposed system can be used as an online platform where customers can send commands to actuators and monitor system readings via the internet. Intranet mode, for local applications, where an MQTT server is configured to control internal network components. Besides, the system was implemented meeting the

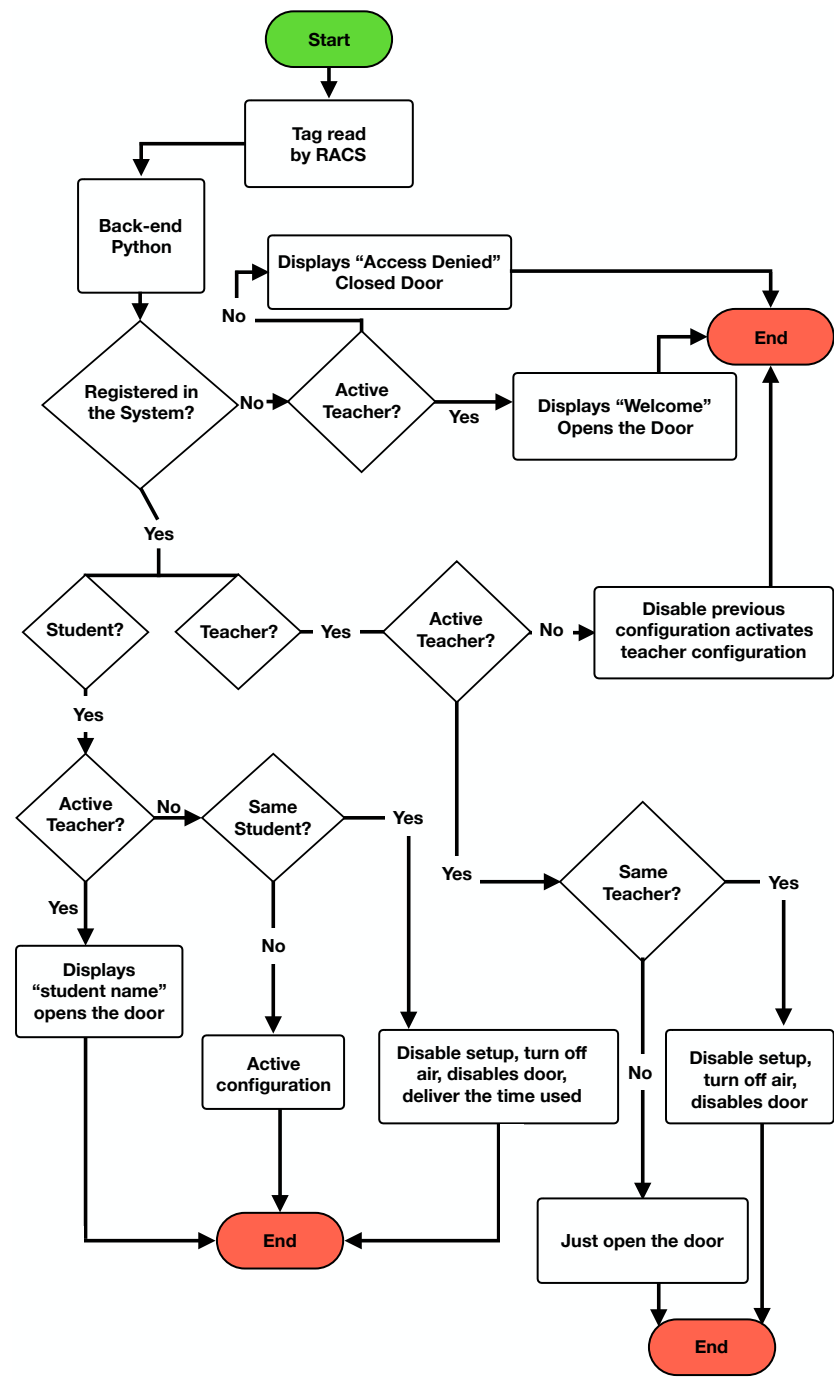

Fig. 9. System flowchart.

requirements of the MVC (model, view, and controller) design standard. We added PDO (PHP Data Object) technology to ensure greater security of user data. Fig 10 shows the registration screen of a certain user, where it is possible to register the personal data of the client. It is possible to define a profile for this same screen, where the temperature is previously defined. Port Status, to inform if the user is allowed to access the room. Air Status, where it is set if the customer can use the device. In the time field, it is set to when the entry will be available to a visiting user, for example.

Only the system administrator has the credentials to add, edit, and delete users on the system, while other system users can view similar ones and edit students. This functionality was added due to the need detected in use cases where the student requests authorization from the teacher to have access to the classroom for a certain moment.

Was used the PHP programming language, along with HTML and Bootstrap to implement the Web system. To persist the data we used the MYSQL database. Finally, to keep the system available, 


$\begin{array}{llll}\text { SmartRoom } & \text { Register } \nabla & \text { Users } \nabla & \text { Exit }\end{array}$

\section{Employee Registration}

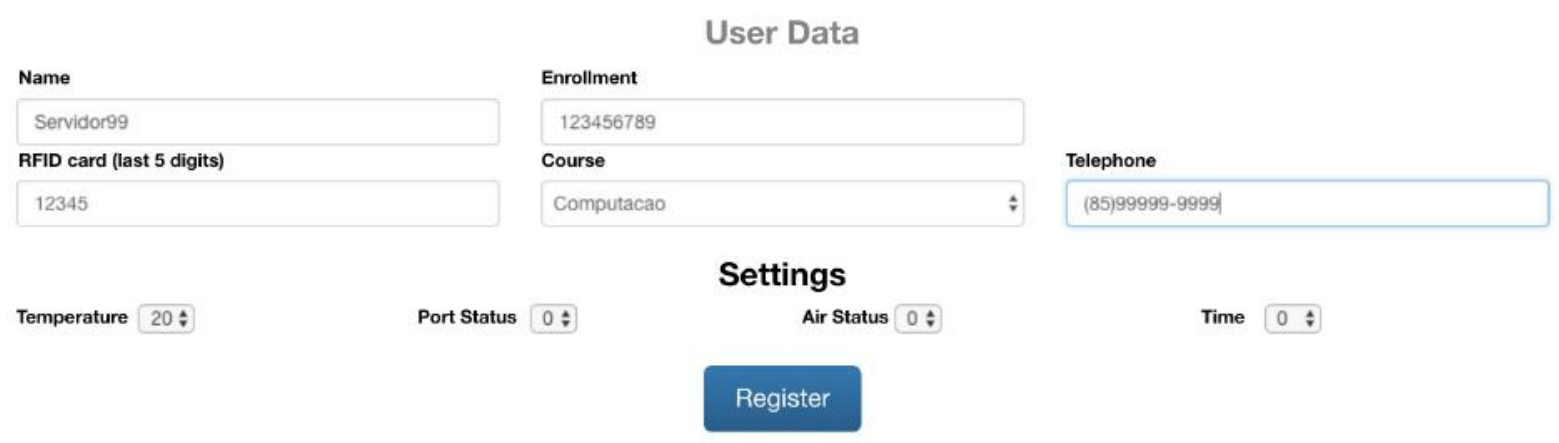

Fig. 10. Employees registration screen.

we used the APACHE server for local configuration.

\section{BACK-END}

All system logic is handled by a script developed in Python. The operating principle follows in this order: each user's unique id of the card is identified by the RFID reader of the RACS system, the tag numbering is sent to the back-end that queries the pertinent information the tag received in the MYSQL database. After the information is requested, a JSON is assembled and published using the MQTT protocol where both the RACS and IACC systems are enrolled.

The RACS system receives all the information regarding the tag and treats the response to obtain the information that will be necessary for room access. The IACC system also receives the information and removes the control profile information from the room air conditioner and temperature controller.

\section{FINAL CONSIDERATIONS}

The developed platform allows managing air conditioners through RFID tag recognition at a low cost. Besides, it promotes greater convenience and speed when turning on / off air conditioners. The vast majority of electronic devices, especially air conditioners, do not yet have automated factory control [15]. Therefore, by creating simple and inexpensive integrated circuits, you can add new automatic features.

As future work, it is sought:

(1) Deploy a multiplatform application to management, to facilitate access to users who have mobile devices.
(2) Expand electronic devices and controlled environments that require strict energy expenditure control, as it allows one or more equipment to be controlled simultaneously, as long as they are organized in the same logical group.

(3) Create a mesh network with esp32 microcontrollers, so that they operate functionally in environments without an internet connection, in addition to implementing a network of sensors that communicate with each other.

(4) Feed a database of air conditioning signals.

Finally, a study to apply the technology presented here should be carried out to measure, even from a scientific perspective, the financial impact of its implementation and the generated economy. For this, it is intended to carry out a project together with a public educational institution.

\section{REFERENCES}

[1] Sangreman A. and Camanho T. Rfid, 2017.

[2] ABESCO. Energy waste reaches $\$ 61.7$ billion in three years, 2017.

[3] T. Almeida. How to calculate air conditioner power consumption, 2016.

[4] P. H. M. Araújo. Rfid access control using the internet of things principle. Escola Regional de Informática do Piauí (ERIPI), 2016.

[5] Pedro Hericson Machado Araújo, Sandro César Silveira Jucá, Diego Lima, Carvalho Gonçalves, Vinícius Ferreira da Silva, Renata Imaculada Soares, and Solonildo Almeida da Silva Pereira. Cloud-based rfid access control using lightweight messaging protocol, 2019.

[6] L. G. Bertelli. Energy waste, 2019.

[7] Bhadrachalam, Lakshmi and Chalasani, Suresh and Boppana, Rajendra V. Impact of RFID Technology on Economic Order 
Quantity Models. In 2009 3rd Annual IEEE Systems Conference, pages 327-332. IEEE, 2009.

[8] Caio Bolzani. Demystifying home automation. Polytechnic School of the University of Sao Paulo, 2007.

[9] Carsten Bormann, Angelo P Castellani, and Zach Shelby. Coap: An application protocol for billions of tiny internet nodes. IEEE Internet Computing, (2):62-67, 2012.

[10] Newton BRAGA. How rfid - radio frequency identification works, 2015

[11] Shi-Cho Cha, Jyun-Fu Chen, Chunhua Su, and Kuo-Hui Yeh. A blockchain connected gateway for ble-based devices in the internet of things. IEEE Access, 6:24639-24649, 2018.

[12] Leonardo da Silva Costa et al. Plataforma iot para automação de controle ir não invasivo aplicado em ar-condicionado. Encontro Unificado de Computação (ENUCOMP), 2017.

[13] National Research Council et al. Committee on radio frequency identification technologies,(2004). In Radio Frequency Identification Technologies: a Workshop Summary.

[14] da Silva, Alana Marques Honorato Lopes and Ramos, Ana Carolina Pereira and de Oliveira, Maisa Bolotari and Jardim, Michely Rodrigues and Pontes, Ana Valéria Vargas. Quality of Life at Work: The Case of a Printing Industry in Juiz de Fora - MG. In Anais-CAT-Congresso de Administração e Tecnologia, volume 2, pages 21-21, 2016.

[15] Tiago José de Araújo Ribeiro and Sérgio Campello Oliveira. Designing a gateway for home automation. Engineering and Applied Research Journal, 1(1), 2016

[16] EPE. Use of air conditioning in the brazilian residential sector: Perspectives and contributions to the advance in energy efficiency, 2018.

[17] João Figueiredo, Miguel Ayala Botto, and Manuel Rijo. Scada system with predictive controller applied to irrigation canals. Control Engineering Practice, 21(6):870-886, 2013.

[18] Glover, Bill and Bhatt, Himanshu and others. RFID Fundamentals. Rio de Janeiro, pages 1-17, 2007.

[19] Kawade A. Prabhune S. Kulkarni, R. and A. R. Dhakne. Web based monitoring of sensor data for smart cities. International Journal of Engineering Science, 7(6):1-3, 62017.

[20] J. C. Lima. Feasibility study of low cost home automation deployment, 2015. (Electrical Engineering Monograph), URCAMP (Cariacica College), Cariacica, Brasil.

[21] Adeline Mercier. Universal infrared adapter for air conditioners, 2015.

[22] Derek Molloy. Exploring raspberry PI. Wiley Online Library, 2016.

[23] MQTT.org. What is mqtt?, 2013.

[24] Rajeev Piyare. Internet of things: ubiquitous home control and monitoring system using android based smart phone. International journal of Internet of Things, 2(1):5-11, 2013.

[25] Sensinode. Hartke K. Bormann C. Shelby, Z. and U. B. TZI. Constrained application protocol (coap) draft-ietf-core-coap17, 2013.

[26] Dinesh Thangavel, Xiaoping Ma, Alvin Valera, Hwee-Xian Tan, and Colin Keng-Yan Tan. Performance evaluation of mqtt and coap via a common middleware. In 2014 IEEE Ninth International Conference on Intelligent Sensors, Sensor Networks and Information Processing (ISSNIP), pages 16. IEEE, 2014.
[27] ALEXANDRE VICENZI. BUSTRACKER: Transit Tracking System, 2015. Monografy (Bachelor of Computer Science), FURB (Regional University of Blumenau), Blumenau, Brazil.

[28] Yanjun Zuo. Survivable rfid systems: Issues, challenges, and techniques. IEEE Transactions on Systems, Man, and Cybernetics, Part C (Applications and Reviews), 40(4):406-418, 2010. 